\title{
EFEITO DA ÉPOCA DO PARTO, IDADE MATERNA E SEXO SOBRE O DESEMPENHO PRÉ-DESMAMA DE BEZERROS NELORE (Bos taurus indicus), CRIADOS EXTENSIVAMENTE NO CENTRO-OESTE DO BRASIL
}

\section{(Effect of calving time, maternal age and sex on the performance pre-weaning of Nelore (Bos taurus indicus) calves extensively raised in Middle West of Brazil)}

\author{
VIU, M.A.O.'; LOPES, D.T.2; GAMBARINI, M.L. ${ }^{3}$; OLIVEIRA FILHO, B.D. ${ }^{3}$; \\ FERRAZ, H.T.'; MAGNABOSCO, C.U. ${ }^{4}$; VIU, A.F.M. ${ }^{5}$
}

\begin{abstract}
'Professor da Escola de Veterinária - UFG/Jataí, Laboratório de Reprodução Animal - (64) 3631-1510; ${ }^{2}$ Alunos de Pós-Graduação em Ciência Animal - EV/UFG. Bolsista CNPq/CAPES;

${ }^{3}$ Professores de Reprodução Animal - EV/UFG/Goiânia;

${ }^{4}$ Pesquisador da EMBRAPA Cerrados - Bolsista CNPq; ${ }^{5}$ Professora da Escola de Ciências Biológicas - UFG/Jataí.
\end{abstract}

\begin{abstract}
RESUMO - Foram utilizados registros de peso ao nascimento (PN), peso à desmama (PD) e ganho médio diário (GMD) de 2.973 bezerros Nelore nascidos de 1992 a 2003, em um sistema de produção extensivo, no Sudoeste de Goiás, de clima tropical e estações seca e chuvosa bem definidas, com o objetivo de determinar a influência ambiental, representada pela época do parto, idade materna e sexo sobre o desempenho prédesmama em sistemas de produção comerciais sem estação de monta definida. Realizou-se a análise de variância por meio do procedimento GLM (SAS, 2000), usando-se a opção Random, que trata o efeito materno como aleatório. O modelo matemático continha os efeitos fixos de ano do parto, estação do parto, sexo do bezerro, idade do bezerro aninhado em sexo, idade da matriz (em classes), além do efeito linear e quadrático da idade da vaca ao parto como co-variável. As médias de PN, PD e GMD encontradas foram, respectivamente, $29,49 \pm 3,45 ; 183,62 \pm 21,62$ e 0,568 $\pm 0,088 \mathrm{~kg}$. Conclui-se que, para a região estudada, os efeitos ambientais, representados pela estação do nascimento, idade da mãe e sexo influenciaram diretamente o desempenho dos bezerros na fase pré-desmama.
\end{abstract}

Palavras-chave: efeito ambiental; efeito materno; gado de corte; ponderal.

ABSTRACT - Birth weight (PN), weaning weight (PD) and daily weight gain (GMD) of 2.973 Nelore calves born from 1992 until 2003 in an extensive cattle production system in Southwest of Goiás - Brazil, characterized by tropical climate and rainy and dry well defined seasons, aiming to verify the influence of environment, represented by time of birth, cow age and sex on the pre weaning performance in commercial production systems without defined breeding season. Variance analysis was used, through the GLM procedure applying the Random option and using maternal effect as an aleatory parameter. The mathematical model contained birth year, calving season, calf sex, calf age nested in sex, cow age (in classes), besides the lineal and quadratic effect of calving age as co-variable. BW, WW and DWG averages were, respectively, 29, 49 \pm 3 , 45; $183,62 \pm 21,62$; and $0,568 \pm 0,088 \mathrm{~kg}$. In conclusion, for the studied region environment effects, as time of parturition, maternal age and sex influence directly the pre weaning performance of Nelore calves.

Key-words: beef cattle; environmental effect; maternal effects.

\section{Introdução}

Na bovinocultura de corte o rápido desenvolvimento é uma característica desejável, pois animais que têm maior velocidade de crescimento atingem o peso de abate mais precocemente. Por isso, os ganhos médios diários, que são características de média à alta herdabilidade, devem ser pesquisados e incluídos em programas de seleção e melhoramento genético (SARMENTO et al., 2003). Um dos primeiros indicativos do potencial genético para o ganho de peso de um animal é o desempenho dos bezerros do nascimento até a desmama. No entanto, esta fase é muito influenciada pela habilidade materna da vaca, pelo genótipo do bezerro e pelo ambiente no qual mãe e filho são criados. Fatores ambientais como o ano e mês de nascimento, o sexo do bezerro e a idade da vaca ao parto, são importantes fontes de variação sobre as características de crescimento (SILVEIRA et al., 2004).

$\mathrm{Na}$ região Centro-Oeste do Brasil, as estações chuvosa e seca bem definidas determinam sasonalidade na produção de forragem, e a conseqüente necessidade do desenvolvimento de estratégias de manejo que favoreçam a concentração de partos em épocas mais favoráveis à amamentação e à desmama, como a definição de uma estação de monta, que elimina ou reduz a necessidade de alguma forma de suplementação, ou a distribuição de partos durante todo o ano, porém com oferta de suplemento durante os meses de menor disponibilidade de alimento (VALLE, 1996). Neste último, os efeitos ambientais, especialmente aqueles relacionados com a mãe serão mais evidentes, e ignorá-los pode resultar em perdas 
VIU, M.A.O. et al.

na avaliação dos parâmetros de produtividade do rebanho estudado (MEYER, 1992).

O objetivo deste trabalho foi avaliar a influência de fatores ambientais como estação e ano de nascimento, sexo do bezerro e idade da fêmea ao parto sobre o peso ao nascimento, à desmama e o ganho médio diário de bezerros Nelore criados extensivamente na região sudoeste de Goiás, sem estação de monta definida.

\section{Material e Métodos}

Dados de 2.973 bezerros PO Nelore (1992 a 2003), filhos de 849 fêmeas de um sistema de produção sem estação de monta, no sudoeste de Goiás, de clima tropical e estações chuvosa e seca bem definidas foram estudados. As fêmeas foram mantidas em pastagens de Brachiaria brizantha Hochst ex. A. Rich Stapf adequadamente manejadas, recebendo sal mineral ad libitum nas chuvas e concentrado protéico-energético (20\% proteína bruta, 70\% NDT) na seca, sem nenhum manejo nutricional diferenciado para bezerros prédesmama. Os bezerros eram pesados no dia do parto e aos oito meses de idade, na desmama. Os dados reprodutivos e peso ao nascimento $(\mathrm{PN})$, à desmama
(PD) e ganho médio diário (GMD) dos bezerros obtidos nas fichas de anotação íntegras geraram o arquivo geral, descartando-se animais com PD acima de $240 \mathrm{~kg}$ e idade maior que 320 dias. As épocas de parto foram agrupadas em: janeiro, fevereiro e março (E1); abril, maio e junho (E2); julho, agosto e setembro (E3); e outubro, novembro e dezembro (E4). A idade da fêmea ao parto foi dividida em 10 classes, de quatro a quatorze anos. Utilizou-se os procedimentos do Statistical Analysis System v. 8.0 (SAS, 2000); procedeu-se a análise de variância por meio do General Linear Model Procedure, com opção RANDOM, para tratar o efeito materno como aleatório (EAM). O modelo matemático continha os efeitos fixos de ano do parto, estação do parto, sexo do bezerro, idade do bezerro aninhado em sexo, idade da matriz (em classes), e efeito linear e quadrático da idade da vaca ao parto (IVP) como co-variável.

\section{Resultados e Discussão}

O PN médio foi de 29,49 $\pm 3,45 \mathrm{~kg}$ (TABELA 1), com influência do ano $(P<0,01)$ e mês do parto $(\mathrm{P}<0,01)$.

TABELA 1 - MÉDIAS, DESVIOS-PADRÃO E COEFICIENTES DE VARIAÇÃO GERAIS E POR SEXO DAS CARACTERÍSTICAS PESO AO NASCIMENTO (PN), PESO À DESMAMA (PD) E GANHO MÉDIO DIÁRIO (GMD), JATAÍ-GO, 2006.

\begin{tabular}{|c|c|c|c|c|c|c|}
\hline \multirow[b]{2}{*}{ Variáveis } & \multicolumn{3}{|c|}{ Machos } & \multicolumn{3}{|c|}{ Fêmeas } \\
\hline & $\mathrm{N}$ & $\mu \pm D P$ & $\mathrm{CV}$ & $\mathrm{N}$ & $\mu \pm D P$ & $\mathrm{CV}$ \\
\hline Peso ao Nascimento & 1476 & $30,45 \pm 3,42^{a}$ & 11,23 & 1497 & $28,54 \pm 3,20^{b}$ & 11,22 \\
\hline Peso à Desmama & 1476 & $189,05 \pm 21,85^{a}$ & 11,56 & 1497 & $178,26 \pm 20,00^{b}$ & 11,22 \\
\hline Ganho Médio Diário & 1476 & $0,5904 \pm 0,09^{a}$ & 15,80 & 1497 & $0,5473 \pm 0,08^{b}$ & 14,37 \\
\hline \multicolumn{7}{|c|}{ Médias, Desvios Padrão e Coeficientes Gerais } \\
\hline Variáveis & & $\mathrm{N}$ & \multicolumn{2}{|c|}{$\mu \pm D P$} & \multicolumn{2}{|c|}{ CV } \\
\hline Peso ao Nascimento & \multicolumn{2}{|r|}{2973} & \multicolumn{2}{|c|}{$29,4900 \pm 3,4467$} & \multicolumn{2}{|c|}{11,6877} \\
\hline Peso à Desmama & \multicolumn{2}{|r|}{2973} & \multicolumn{2}{|c|}{$183,6152 \pm 21,6199$} & \multicolumn{2}{|c|}{11,7746} \\
\hline Ganho Médio Diário & \multicolumn{2}{|r|}{2973} & \multicolumn{2}{|c|}{$0,5687 \pm 0,0889$} & \multicolumn{2}{|c|}{15,6270} \\
\hline
\end{tabular}

Letras diferentes na mesma linha indicam diferença (1\% de probabilidade) pelo teste $t$.

ALENCAR et al. (1999) notaram efeito do ano de nascimento mas não do mês sobre PN, relatando que a diferença de um ano para outro se deve às variações na disponibilidade de alimentos e no manejo. Para FERRELL e REYNOLDS (1992), o crescimento fetal bovino é elevado no terço final da gestação, quando ocorre a maior parte da deposição tecidual, com aumento do metabolismo materno; do total de dispêndio de energia na gestação, cerca de metade é atribuída ao metabolismo do útero gravídico, e um quarto ao feto propriamente dito. Aqui o efeito do mês do parto foi evidente; embora ocorram durante todo o ano, houve maior número na E3 e E4, mostrando que as matrizes que passaram o terço final da gestação durante 0 período de maior disponibilidade quantitativa e qualitativa de alimento, foram capazes de suprir mais adequadamente suas necessidades nutricionais $e$ manter o crescimento fetal, refletido no maior PN. A ausencia de influência da interação ano-mês-sexo indica que as condições climáticas da região, o manejo adotado e o dimorfismo sexual não afetaram as diferenças de PN, discordando de RIBEIRO et al. (2001), que verificaram influência $(P<001)$ desta interação no PN de animais Nelore, ou dos relatos de ALENCAR et al. (1999), com animais Canchim x Nelore.

O PD médio foi 183,62 $\pm 21,62 \mathrm{~kg}$ (TABELA 1), com influencia $(P<0,01)$ do ano e estação do parto. ALENCAR et al. (1999) descreveram maiores PD‘s para animais nascidos entre os meses de junho e setembro, no final da estação seca. Para SILVEIRA et al. (2004), mesmo com menor disponibilidade de forragem para a mãe, afetando a produção de leite, a exigência do bezerro é menor em função do peso baixo. Com o crescimento eleva-se o consumo de leite, mas há aumento da produção porque as alterações ambientais aumentam a disponibilidade de forragem até o final do 
Efeito da época do parto, idade materna e sexo sobre o desempenho pré-desmama de bezerros Nelore...

período das águas, quando ocorre a desmama (TORAL et al., 2004). Após o pico de lactação os bezerros, com cerca de três meses de idade, são ruminantes e não dependem da amamentação.

A IVP e as interações entre ano/mês do parto e sexo do bezerro não afetaram o PN (TABELA 2). Para MORRIS et al. (2006), vacas jovens produzem bezerros mais leves, pois sofrem mais o efeito ambiental durante a gestação, e por isso devem ser mantidas separadas das vacas velhas, evitando a competição pelo alimento. MARTINS et al. (2000) não verificaram a influência da idade da vaca sobre o PN em rebanhos Nelore no Maranhão. Para MACMANUS et al. (2002) isso ocorre pela pressão de seleção, restando animais superiores, com menores IDPs, maior PD e à manutenção de filhas precoces e boas reprodutoras.

O EAM foi significativo $(P<0,01)$ para $P N, P D$ e $G M D$ (TABELAS 2,3 e 4), concordando com SARMENTO et al. (2003), e reflete efeitos genéticos transmitidos aos bezerros e maternos, considerados de origem ambiental os mesmos.

TABELA 2 - RESUMO DA ANÁLISE DE VARIÂNCIA PARA PESO AO NASCIMENTO, JATAÍ-GO, 2006.

\begin{tabular}{lccccc}
\hline \multicolumn{1}{c}{ Fontes de Variação } & $\mathrm{GL}$ & $\mathrm{SQ}$ & $\mathrm{QM}$ & $\mathrm{F}$ & $\mathrm{Pr}>\mathrm{F}$ \\
\hline Efeito aleatório da reprodutiz & 848 & 15920,19 & 18,77 & 2,42 & 0,0001 \\
Mês do parto (MP) & 11 & 167,02 & 15,18 & 1,96 & 0,0290 \\
Ano do parto (AN) & 11 & 629,80 & 57,25 & 7,38 & 0,0001 \\
Sexo (SX) & 1 & 1864,64 & 1864,64 & 240,32 & 0,0001 \\
MP*AN & 102 & 1079,15 & 10,58 & 1,36 & 0,0107 \\
NA*MP*SX & 106 & 925,19 & 8,73 & 1,12 & 0,1867 \\
Idade da vaca (linear) & 1 & 6,60 & 6,60 & 0,85 & 0,3566 \\
Idade da vaca (Quadrático) & 1 & 14,59 & 14,59 & 1,88 & 0,1705 \\
Idade da vaca em classes & 9 & 97,48 & 10,83 & 1,40 & 0,1843 \\
Resíduo & 1882 & 14602 & 7,76 & & \\
\hline Coeficiente de determinação & \multicolumn{5}{c}{0,5864} \\
\hline
\end{tabular}

TABELA 3 - RESUMO DA ANÁLISE DE VARIÂNCIA PARA PESO À DESMAMA, JATAÍ-GO, 2006.

\begin{tabular}{lccccc}
\hline \multicolumn{1}{c}{ Fontes de Variação } & $\mathrm{GL}$ & $\mathrm{SQ}$ & $\mathrm{QM}$ & $\mathrm{F}$ & $\mathrm{Pr}>\mathrm{F}$ \\
\hline Efeito aleatório da reprodutiz & 848 & 637311,19 & 751,55 & 2,72 & 0,0001 \\
Ano do parto (AN) & 11 & 15243,43 & 1385,77 & 5,02 & 0,0001 \\
Estação do parto (EST) & 3 & 6009,53 & 2003,18 & 7,26 & 0,0001 \\
Sexo (SX) & 1 & 64929,50 & 64929,50 & 235,30 & 0,0001 \\
NA*EST & 32 & 38705,90 & 1209,56 & 4,38 & 0,0001 \\
Idade (Sexo) & 2 & 43428,52 & 21714,26 & 78,69 & 0,0001 \\
NA*EST*SX & 45 & 13782,38 & 306,28 & 1,11 & 0,2861 \\
Idade da vaca (linear) & 1 & 2607,96 & 2607,96 & 9,45 & 0,0021 \\
Idade da vaca (Quadrático) & 1 & 4910,45 & 4910,45 & 17,80 & 0,0001 \\
Idade da vaca em classes & 9 & 5120,30 & 568,92 & 2,06 & 0,0298 \\
Resíduo & 2019 & 557122,63 & 275,94 & & \\
\hline Coeficiente de determinação & & & & \\
\hline
\end{tabular}

TABELA 4 - RESUMO DA ANÁLISE DE VARIÂNCIA PARA GANHO MÉDIO DIÁRIO, DO NASCIMENTO À DESMAMA, JATAÍ-GO, 2006.

\begin{tabular}{|c|c|c|c|c|c|}
\hline Fontes de Variação & $\mathrm{GL}$ & SQ & QM & $\mathrm{F}$ & $\mathrm{Pr}>\mathrm{F}$ \\
\hline Efeito aleatório da reprodutiz & 848 & 10,8734 & 0,0128 & 2,77 & 0,0001 \\
\hline Ano do parto (AN) & 11 & 0,1324 & 0,0120 & 2,60 & 0,0028 \\
\hline Mês do parto (MP) & 11 & 0,3199 & 0,0290 & 6,28 & 0,0001 \\
\hline Sexo (SX) & 1 & 0,9383 & 0,9383 & 202,51 & 0,0001 \\
\hline$M P^{*} A N$ & 102 & 1,5965 & 0,0157 & 3,38 & 0,0001 \\
\hline$N A^{*} M P^{*} S X$ & 106 & 0,6504 & 0,0061 & 1,32 & 0,0172 \\
\hline Idade da vaca (linear) & 1 & 0,0174 & 0,0174 & 3,76 & 0,0525 \\
\hline Idade da vaca (Quadrático) & 1 & 0,1942 & 0,1942 & 41,92 & 0,0001 \\
\hline Idade da vaca em classes & 9 & 0,0285 & 0,0032 & 0,68 & 0,7256 \\
\hline Resíduo & 1882 & 8,7200 & 0,0046 & & \\
\hline Coeficiente de determinação & & & 0,6285 & & \\
\hline
\end{tabular}


VIU, M.A.O. et al.

A idade da vaca ao parto teve efeito linear e quadrático $(P<0,01)$ sobre $P D$. Para isto contribui o grau de maturidade da matriz; vacas adultas, em geral, desmamam bezerros mais pesados que as mais novas ou velhas (MUNIZ e QUEIROZ,1998). O efeito linear pode ser atribuído à manutenção de vacas que desmamam bezerros mais pesados, como preconizado por MACMANUS et al. (2002). A interação ano-estação do parto-sexo do bezerro não influenciou o PD (TABELA 3). Já RIBEIRO et al. (2001), na Paraíba, verificaram este efeito para animais Nelore.

O GMD foi $0,568 \pm 0,088 \mathrm{~kg}$ (TABELA 1), próximo aos descritos por LÔBO et al. (1995), BIFFANI (1997) e MARTINS FILHO et al. (1997), com influência significativa do ano e mês do parto $(\mathrm{P}<0,01)$, reforçando que partos no período de maior disponibilidade de alimento para a vaca e sua cria favorecem o desenvolvimento precoce do bezerro (BIFFANI,1997; ALENCAR et al. 1999). A interação entre ano-mês de nascimento-sexo do bezerro influenciou $(P<0,05) 0$ GMD, como descrito por RIBEIRO et al. (2001) e SARMENTO et al. (2003). Apenas o efeito quadrático da co-variável idade da vaca ao parto interferiu $(P<0,01)$ no GMD (TABELA 4), sugerindo que matrizes mais novas ou velhas desmamam bezerros mais leves (MUNIZ e QUEIROZ, 1998). Na fêmea jovem a partição de nutrientes implica na continuidade do processo de crescimento concomitante com lactação e mantença, e na fêmea velha ocorre a redução na habilidade em arcar com as necessidades nutricionais e outros fatores de estresse associados ao envelhecimento (MORRIS et al., 2006).

$O$ sexo do bezerro influenciou $(P<0,01) P N, P D$ e GMD, que foram maiores nos machos que nas fêmeas (TABELA 1), mostrando a contribuição do dimorfismo sexual para a diferença de pesos. ALENCAR et al. (1999) verificaram, em bezerros Nelore x Canchim, GMD's maiores para machos, com médias superiores às verificadas nesse estudo. Já MARTINS et al. (2000) não relataram influência do sexo sobre $\mathrm{PN}$ de bezerros Nelore, no Maranhão. KANUYA et al. (2006), estudando esta característica em rebanhos zebuínos mantidos em regiões climáticas semelhantes àquela do presente estudo, verificaram forte influência do sexo sobre o GMD.

\section{Conclusões}

$\mathrm{Na}$ região estudada efeitos ambientais tais como estação do nascimento, idade da mãe e sexo influenciaram diretamente o desempenho dos bezerros na fase pré-desmama, e devem ser considerados para a tomada de decisões relacionadas à definição de estação de nascimentos e, conseqüentemente, de desmama, para obtenção de melhores índices de produtividade no rebanho, sem onerar o sistema de produção.

\section{Referências}

ALENCAR, M.M.; OLIVEIRA, M.C.S.; BARBOSA, P.F. Causas da variação de características de crescimento de bovinos cruzados Canchim x Nelore. Revista Brasileira de Zootecnia, v.28, n.4, p.687-692, 1999.

BIFFANI, S. Influência de fatores ambientais sobre o crescimento de bovinos da raça Nelore e estimativa de parâmetros genéticos pelos métodos Henderson 3 e REML. Fortaleza: Universidade Federal do Ceará, 1997. 121p. Dissertação (Mestrado em Zootecnia - Universidade Federal do Ceará, 1997).

FERRELL, C.L.; REYNOLDS. L.P. Uterine and umbilical blood flows and net nutrient uptake by fetuses and uteroplacental tissues of cows gravid with either single or twin fetuses. Journal of Animal Science, 70, p.426-433. 1992.

KANUYA, N.L.; MATIKO, M.K.; KESSY, B.M.; MCGONGO, F.O.; ROPSTAD, E.; REKSEN, O. A study on reproductive performance and related factors os zebu cows in pastoral herds in a semi-arid area of Tanzania. Theriogenology, v.65, p.1859-1874, 2006.

LÔBO, R.B.; BORJAS, A. De los, R.; BEZERRA, L.A. ; PIMENTA, C.M.S.J.; SILVA, J.A.V.; TAVARES, H.F. Parâmetros fenotípicos e genéticos de pesos e perímetro escrotal às idades-padrão em animais da raça Nelore. In: REUNIÃO ANUAL DA SOCIEDADE BRASILEIRA DE ZOOTECNIA, 32., 1995, Brasília. Anais...Brasília: Sociedade Brasileira de Zootecnia, 1995. p.625-627.

MARTINS, G.A.; MARTINS FILHO, R.; LIMA, F.A.M.; LÔBO, R.N.B. Influência de fatores genéticos e de meio sobre o crescimento de bovinos da raça Nelore no Estado do Maranhão. Revista Brasileira de Zootecnia, v.29, n.1, p.103-107, 2000.

MARTINS FILHO, R.; LÔBO, R.B.; LIMA, F.A.M. Parâmetros genéticos e fenotípicos de pesos e ganhos em pesos de bovinos zebus no Estado do Ceará. In: REUNIÃO ANUAL DA SOCIEDADE BRASILEIRA DE ZOOTECNIA, 34., 1997, Juiz de Fora. Anais...Juiz de Fora: Sociedade Brasileira de Zootecnia, 1997. p.248250.

MACMANUS, C.; SAUERESSING, M.G.; FALCÃO, R.A.; SERRANO, G.; MARCELINO, K.R.A.; PALUDO, G.R. Componentes Reprodutivos e Produtivos no Rebanho de Corte da Embrapa Cerrados. Revista Brasileira de Zootecnia, v.31, n.2, p.648-657, 2002.

MEYER, K. Variances components due to direct and maternal effects for growth traits of Australian beef cattle. Livestock Production Science, v.31, p.179240, 1992.

MORRIS, S.T.; MOREL, P.C.H.; KENYON, P.R. The effect of individual live weight and condition of beef cows on their reproductive performance and birth and weaning weights of calves. New Zealand Veterinary Journal, v.54, n.2, p.96-100, 2006. 
Efeito da época do parto, idade materna e sexo sobre o desempenho pré-desmama de bezerros Nelore...

MUNIZ, C.A.S.D.; QUEIROZ, S.A. Avaliação do peso à desmama e do ganho médio de peso de bezerros cruzados, no Estado do Mato Grosso do Sul. Revista Brasileira de Zootecnia, v.27, n.3, p.504-512, 1998.

RIBEIRO, M.N.; PIMENTA FILHO, E.C.; MARTINS, G.A.; SARMENTO, J.R.L.; MARTINS FILHO, R. Herdabilidade para efeitos direto e materno de características de crescimento de bovinos Nelore no Estado da Paraíba. Revista Brasileira de Zootecnia, v.30, n.4, p.1224-1227, 2001.

SARMENTO, J.L.R.; PIMENTA FILHO, E.C.; RIBEIRO, M.N.; MARTINS FILHO, R. M. Efeitos ambientais e genéticos sobre o ganho em peso diário de bovinos Nelore no Estado da Paraíba. Revista Brasileira de Zootecnia, v.32, n.2, p.325330, 2003.
SAS - User's Guide: statistics. 5.ed. Cary: SAS Institute, 2000. 1028p.

SILVEIRA, J.C.; MACMANUS, C.; MASCIOLI, A.S.; SILVA, L.O.C.; SILVEIRA, A.C.; GARCIA, J.A.S.; LOUVANDINI, $H$. Fatores Ambientais e Parâmetros Genéticos para Características Produtivas e Reprodutivas em um Rebanho Nelore no Estado do Mato Grosso do Sul. Revista Brasileira de Zootecnia, v.33, n.6, p.1432-1444, 2004.

TORAL, F.L.B.; SILVA, L.O.C.; MARTINS, E.N.; GOND, A.; SIMONELLI, S. Interação genótipo $x$ ambiente em características de crescimento de bovinos da raça Nelore no Mato Grosso do Sul. Revista Brasileira de Zootecnia, v.33, n.6, p. 1445-1455, 2004.

VALLE, E.R. do. Estação de monta para bovinos de corte no Brasil Central. Campo Grande: EMBRAPA-CNPGC, 1996. 3p. (EMBRAPA-CNPGC. CNPGC Divulga, 4).

Recebido para publicação: 20/08/2006

Aprovado: $\quad 19 / 12 / 2006$ 